\title{
An Optimization Approach for Removing Blocking Effects in Transform Coding
}

\author{
Shigenobu Minami and Avideh Zakhor
}

\begin{abstract}
One of the drawbacks of the Discrete Cosine Transform (DCT) is visible block boundaries due to coarse quantization of the coefficients. Most restoration techniques for the removing blocking effect are variations of low-pass filtering, and as such, result in unnecessary blurring of the image. In this paper, we propose a new approach for reducing the blocking effect which can be applied to conventional transform coding, such as JPEG standardized coding, without introducing additional information or significant blurring. Our technique exploits the correlation between the intensity values of boundary pixels of two neighboring blocks. Specifically, it is based on the theoretical and empirical observation that under mild assumptions, quantization of the DCT coefficients of two neighboring blocks increases the expected value of the Mean Squared Difference of Slope (MSDS) between the slope across two adjacent blocks, and the average between the boundary slopes of each of the two blocks. The amount of this increase is dependent upon the width of quantization intervals of the transform coeficients. Therefore, among all permissible inverse quantized coefficients, the set which reduces the expected value of this MSDS by an appropriate amount is most likely to decrease the blocking effect. To estimate the set of unquantized coefficients, we solve a constrained quadratic programming problem in which the quantization decision intervals provide upper and lower bound constraints on the coefficients. Our approach is based on the gradient projection method which is motivated by the ordinary method of steepest descent for unconstrained problems. Computer simulations are used to evaluate the performance of the proposed technique. We have shown that from a subjective viewpoint, the blocking effect is less noticeable in our processed images than in the ones using existing filtering techniques.
\end{abstract}

\section{INTRODUCTION}

D ISCRETE COSINE TRANSFORM (DCT) is among the most popular transform techniques for image processing because of its compaction property and relative ease of implementation. One of its drawbacks however, is visible block boundaries due to coarse quantization of the coefficients. This problem becomes especially serious when the DC or near-DC coefficients are coarsely quantized. Therefore, more bits are usually required for the DC or near DC coefficients than for other coefficients.

Manuscript received September 4, 1991; revised November 8, 1994. This work was supported by NSF PYI Grant MIP-90-57466, ONR Young Investigator Award N00014-92-J-1732, California State Program MICRO, Sun Microsystems, and Hewlett Packard. This paper was recommended by Associate Editor J. W. Woods.

S. Minami was with the University of California, Berkeley, CA. He is now with the Information and Communication Systems Laboratory, Toshiba Corp., Hino City, Tokyo, 191 Japan.

A. Zakhor is with the University of California, Berkeley, CA 94720 USA. IEEE Log Number 9410394.
To cope with the blockiness problem without increasing the bit rate, the following approaches have been proposed: (a) Low-Pass filtering on boundary pixels [1], [2]; (b) DPCM-DC approach [3], [4]; (c) AC prediction using neighboring block DC coefficients [3], [4]. Since the blocking effect is primarily due to the inability of the DCT to exploit inter block correlations, most of the above approaches exploit correlations of intensity values in neighboring blocks. The low-pass filtering approach, which reduces high frequency components near the block boundary, has the advantage that it does not require any additional information to be transmitted or any additional operation on the coder side [1], [2]. The biggest drawback to this approach, which is common to most low-pass filtering approaches, is unnecessary blurring of the image. Another widely accepted approach for reducing blocking effect is to apply a Differential Pulse Code Modulation (DPCM) to the DC component of the DCT coefficients [3], [4]. This approach, which is actually implemented in JPEG, reduces the blocking effect caused by coarse quantization of the DC coefficient by exploiting the correlation of DC coefficients in neighboring blocks. However, it requires strict synchronization ${ }^{1}$ between coder and decoder, and cannot improve the blocking effect caused by coarse quantization of non-DC coefficients. Another JPEG approach for the blocking effect is the AC prediction method, where AC components of DCT coefficients are predicted by the DC coefficients in the neighboring blocks. The major drawback of this method is its low performance in reducing blockiness. This is because only a few quantized AC coefficients, which become zero after the quantization, are replaced by predictions of the DC components.

In this paper, we propose a new approach for reducing the blocking effect which can be applied to conventional transform coding without introducing additional information or significant blurring. Our technique which can also be applied in conjunction with traditional techniques such as DPCM on DC, exploits the correlation between intensity values of boundary pixels of two neighboring blocks. Specifically, it is based on the empirical observation that quantization of the DCT coefficients of two neighboring blocks increases the Mean Squared Difference of Slope (MSDS) between the neighboring pixels on their boundaries. Therefore, among all permissible inverse quantized coefficients, the set which minimizes this MSDS is most likely to decrease the blocking effect. This minimization can be formulated as a Quadratic Programming (QP) problem and solved via a variety of quadratic optimization techniques.

\footnotetext{
${ }^{1}$ Strict synchronization requirements are typical of all DPCM schemes.
} 
The outline for the remaining part of this paper is as follows: In Sections II and III, we discuss the blocking effect and address the problems associated with traditional methods. Section IV includes our definition of the MSDS and Section $\mathrm{V}$ formulates our proposed method as a quadratic program problem. In Section VI, we present the experimental results for our approach and Section VII includes our conclusions.

\section{BLOCKING EFFECTS}

Though a variety of coding systems using the DCT has been proposed, block based DCT coding, such as the JPEG method, segment the image into blocks of size $N \times N$, transform them into coefficients and quantize the coefficients. Specifically, the $(i, j)$ th block $b_{i j}$, is transformed into an $N \times N$ dimensional array of DCT coefficients $a_{i j}(u, v)$ :

$$
\begin{gathered}
a_{i j}(u, v)=C(u) C(v) \sum_{k=0}^{N-1} \sum_{l=0}^{N-1} x_{i, j}(k, l) \\
\cdot w_{D C T}(u, k) w_{D C T}(v, l)
\end{gathered}
$$

where $x_{i, j}(k, l)$ is the intensity of the $(k, l)$ th pixel in the $(i, j)$ th block, and $C(u)$ and $w_{D C T}(u, k)$ are operators of the DCT transformation which are defined by

$$
\begin{aligned}
C(u) & =\left\{\begin{array}{cc}
1 / \sqrt{2} & u=0 \\
1 & u \neq 0
\end{array}\right. \\
w_{D C T}(u, k) & =\cos [(2 k+1) \pi u / 2 N] .
\end{aligned}
$$

After this transform, the DCT coefficients are quantized. In our study, JPEG quantization based on a quantization table shown in Fig. 3 was adopted [3], [4]. The entries in the table denote the quantization step size $\Delta(u, v)$ used for the $(u, v)$ th DCT coefficient. In the method, an inverse-quantized coefficient $\tilde{a}_{i j}(u, v)$ is obtained by adding half of the quantization step size $\Delta(u, v)$ to decoded quantization lower limit values. Using the quantized DCT coefficients, a reconstructed pixel $\tilde{x}_{i, j}(k, l)$ is obtained as follows:

$$
\begin{aligned}
\tilde{x}_{i j}(k, l)= & \sum_{u=0}^{N-1} \sum_{v=0}^{N-1} \tilde{a}_{i j}(u, v) \\
& \cdot w_{I D C T}(u, k) w_{I D C T}(v, l)
\end{aligned}
$$

where $w_{I D C T}(u, k)$ is an operator of the Inverse DCT (IDCT) transformation which is defined by

$$
w_{I D C T}(u, k)=C(u) \cos [(2 k+1) \pi u / 2 N] .
$$

An example of the above procedure is shown in Fig. 2, where the original Lena image shown in Fig. 1, is encoded at the rate of $0.27 \mathrm{~b} /$ pixel via JPEG with the quantization table shown in Fig. 3. As evident from the decoded image shown in Fig. 2, the block boundaries are quite noticeable. This blocking effect is one of the drawbacks of block based transform coding.

\section{TRADITIONAL APPROACHES}

One of the traditional approaches to removing the blocking effect is low-pass post-filtering which was proposed by KouHu Tzou [2]. In this approach, an anisotropic low-pass filtering

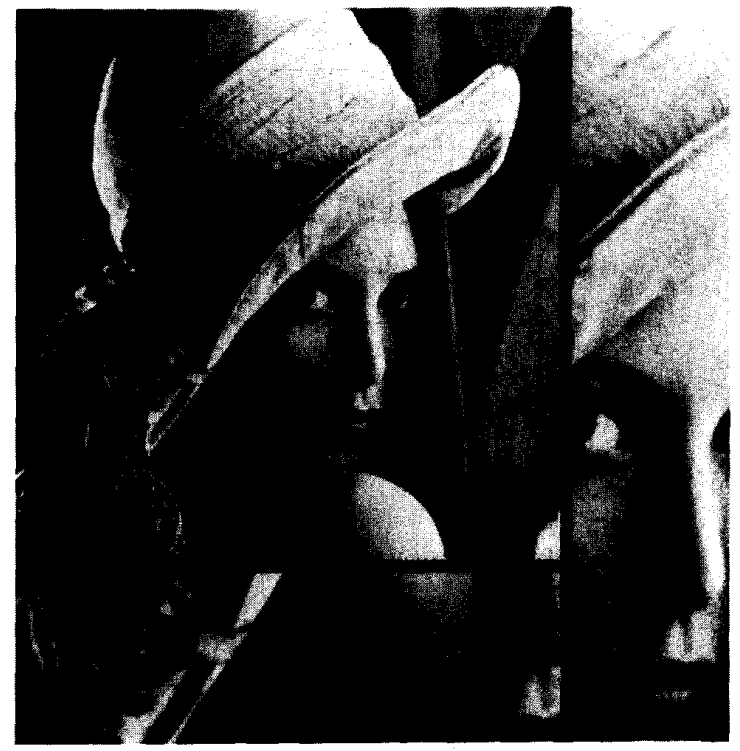

Fig. 1. The original $256 \times 256$ image Lena used in all the experiments in this paper. The intensity of the pixels is quantized to $8 \mathrm{~b}$.

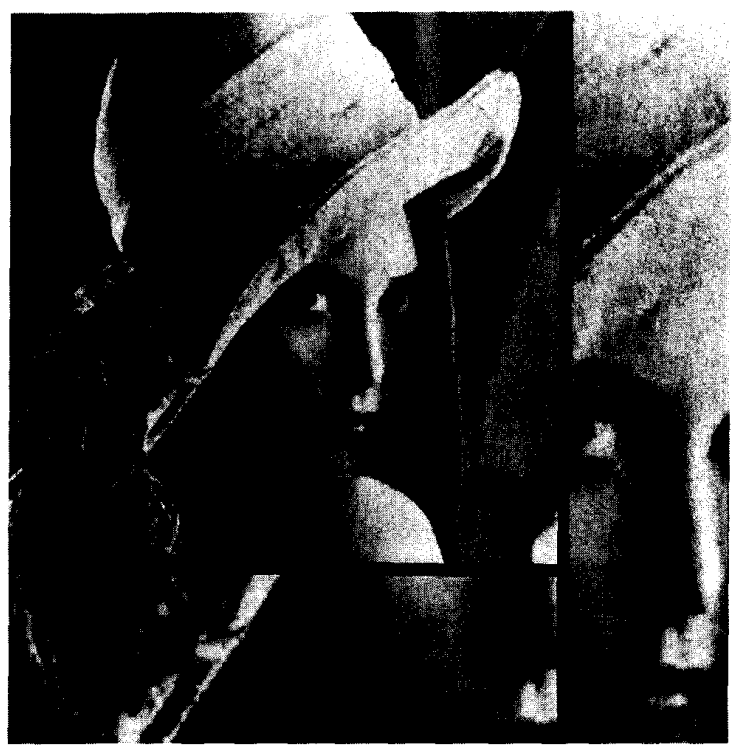

Fig. 2. Significant blocking effects are observed in this picture, where DCT coefficients in each block are quantized at the rate of $0.27 \mathrm{~b} /$ pixel based on JPEG quantization.

is carried out only on the boundary pixels to remove the high frequency part of the visible edges caused by the blocking effect. To keep the sharpness of the decoded image, the major axis of the anisotropic filter is changed by the direction of the block boundaries. An example of this approach is shown in Fig. 4, where low-pass filtering is applied only to the block boundary pixels in the image shown in Fig. 2 . The coefficients of the low-pass filter used for a vertical boundary is $h(0,0)=$ $0.48, h( \pm 1, \pm 1)=.005, h(0, \pm 1)=0.01, h( \pm 1,0)=$ 0.24 . To make clear the quality of the picture, the cheek, 


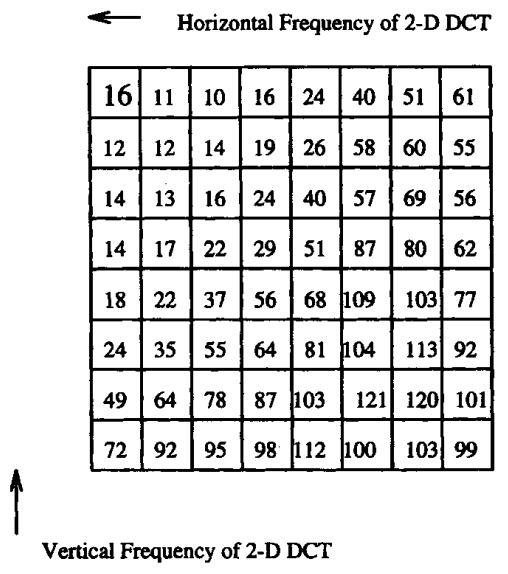

Fig. 3. JPEG quantization table at $0.27 \mathrm{~b}$ /pixel.

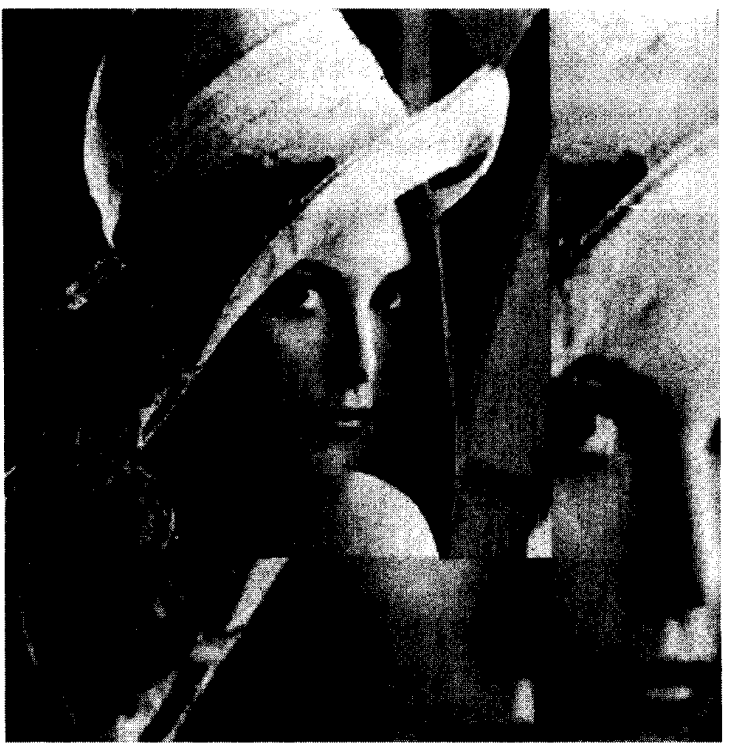

Fig. 4. In this picture anisotropic low-pass filtering is applied to boundary pixels in the JPEG quantized image shown in Fig. 2. We still notice the remaining blocking effects around the cheek area.

eye and nose parts of the picture are enlarged. As evident from Fig. 4, the blocking effect is removed well around Lena's hat area where the original image includes a large number of edges, even though there is some blurring. Also, the blocking effect is still observed in the cheek area, where the original image is smooth. This blocking effect around the cheek area can be removed by making the cut-off frequency of the lowpass filter lower. However, the low cut-off low-pass filter will cause excessive blurring if we do not adopt a complex cutoff frequency control based on the local characteristics of the image.

The AC prediction method used in JPEG is another promising approach to reducing the blocking effect due to the quantization of near DC, DCT coefficients which are called AC components. An example of this method is shown in

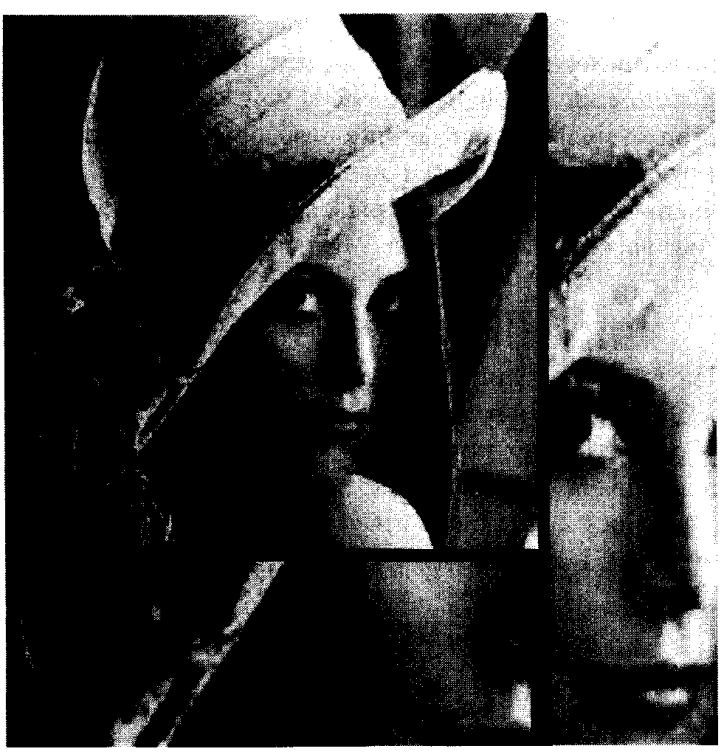

Fig. 5. AC prediction method is applied to the JPEG encoded picture at $0.27 \mathrm{~b}$ /pixel.

Fig. 5 with enlarged pictures, where the original Lena image, shown in Fig. 1, is encoded at the rate of $0.27 \mathrm{~b} /$ pixel by the JPEG method with the AC prediction method. As shown, the blocking effect is still noticeable. This is because the AC prediction is applied only to the zero quantized coefficients and the blocking effect due to the quantization of DC and higher frequency DCT coefficients can not be removed by the method.

To improve the above problems in the traditional approach, we propose a new method in the next section.

\section{MEAN SQuare DifFERENCE OF SLOPE (MSDS)}

As mentioned earlier, the blocking effect results in discontinuities across block boundaries. Based on this observation, we introduce a new criterion called Mean Square Difference of Slope (MSDS). To define this new metric, consider Fig. 6 in which two adjacent horizontal blocks $b_{i, j}$ and $b_{i, j-1}$ are shown. If the coefficients at the two blocks are coarsely quantized, we expect to see a difference in the intensity slope across the block boundary. On the other hand, this abrupt change in intensity slope across the block boundaries of the original unquantized image is rather unlikely. This is because most parts of most natural images can be considered to be smoothly varying and their edges are unlikely to line up with block boundaries.

From the above consideration, it is clear that an appropriate metric to optimize in removing blocking effect, say in Fig. 6 , is to minimize the square of the difference between the slope across two adjacent blocks, and the average between the slope of each of the two blocks close to their boundaries. This quantity is given by

$$
\varepsilon_{F i j}^{2} \triangleq \sum_{k=0}^{N-1}\left[d_{i, j}^{M F}(k)-\bar{d}_{i, j}^{F}(k)\right]^{2}
$$




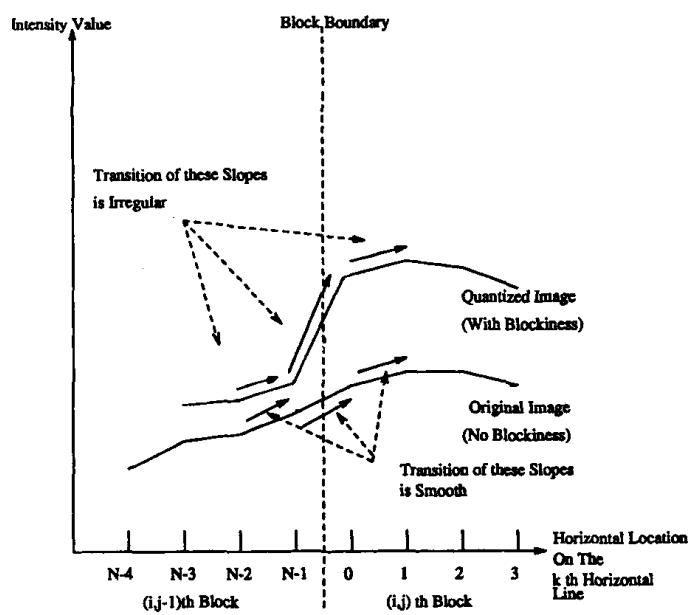

Fig. 6. MSDS is a good measure of blocking effects, because it reflects the discontinuity of the image along block boundaries. MSDS is the mean square difference between actual boundary gradient $d_{i j}^{M F}(k)$ and interpolated gradient $\bar{d}_{i, j}^{F}(k)$ from adjacent slopes along a block boundary.

where $d_{i, j}^{M F}(k)$ is the slope across the boundary between $(i, j)$ and $(i, j-1)$ th blocks defined by

$$
d_{i, j}^{M F}(k) \triangleq x_{i, j}(k, 0)-x_{i, j-1}(k, N-1)
$$

and $\bar{d}_{i, j}^{F}(k)$ is the average between the intensity slopes of blocks $(i, j)$ and $(i, j-1)$ close to their boundaries defined by

$$
\begin{gathered}
\bar{d}_{i, j}^{F}(k) \triangleq\left[d_{i, j-1}^{H}(k, N-1)+d_{i, j}^{H}(k, 1)\right] / 2 \\
d_{i, j-1}^{H}(k, N-1)=x_{i, j-1}(k, N-1)-x_{i, j-1}(k, N-2) \\
d_{i, j}^{H}(k, 1)=x_{i, j}(k, 1)-x_{i, j}(k, 0) .
\end{gathered}
$$

Based on the above, we can expand $\varepsilon_{F i j}^{2}$ in (5) as follows:

$$
\begin{aligned}
\varepsilon_{F i j}^{2}= & \sum_{k=0}^{N-1}\left[\frac{1}{2}\left\langle 3 x_{i, j}(k, 0)-x_{i, j}(k, 1)\right)\right. \\
& \left.-\frac{1}{2}\left(3 x_{i, j-1}(k, N-1)-x_{i, j-1}(k, N-2)\right)\right]^{2} .
\end{aligned}
$$

Note that the above cost function is the energy along the block boundary of the image filtered by the FIR filter with impulse response $\left[\frac{1}{2},-\frac{3}{2}, \frac{3}{2},-\frac{1}{2}\right]$.

We can extend the ideas in the above discussion to two adjacent vertical blocks, or more generally to both horizontal and vertical neighboring blocks. Specifically, if blocks $F$ and $C$ denote the horizontal adjacent blocks to the $(i, j)$ th block, and $B$ and $D$ present the vertical adjacent blocks of the $(i, j)$ th block, then we can consider minimizing total MSDS given by

$$
M S D S \triangleq \varepsilon_{i j}^{2}\left(A_{i, j}\right)=\varepsilon_{F i j}^{2}+\varepsilon_{B i j}^{2}+\varepsilon_{C i j}^{2}+\varepsilon_{D i j}^{2}
$$

where the last three terms in the above sum are defined in a similar way to $\varepsilon_{F i j}^{2}$ already defined in (5), and $A_{i, j}$ is a $N^{2}$ dimensional array which is composed of unquantized coefficients of the $(i, j)$ th block as

$$
A_{i j}=\left[a_{i j}(0), a_{i j}(1) \cdots a_{i, j}(\nu) \cdots a_{i j}\left(N^{2}-1\right)\right]^{T} .
$$

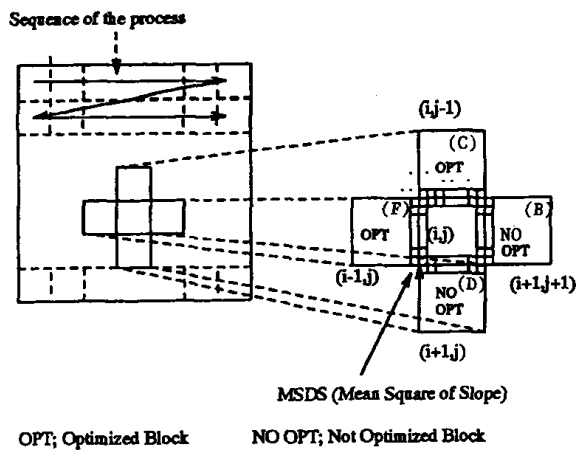

Fig. 7. As shown here, the sequence of the MSDS optimization is from left to right and top to bottom. Therefore, two blocks to the left and above are already optimized and ones to the bottom and to the right are not optimized yet.

As shown in Fig. 6, since the difference between the actual boundary slope and the interpolated slope increases if the image is discontinuous on a block boundary, the probability of the MSDS increase becomes higher after coarse quantization. This is because the blocking effect produces undesirable discontinuities on block boundaries. From this observation, the expected value of the MSDS increase is positive after quantization of DCT coefficients. In Appendix, the expected value of the MSDS is obtained for two cases of quantized and unquantized DCT coefficients. If $\delta M S D S$ denotes the change in $M S D S$ due to quantization, the results in Appendix show that the expected value of $\delta M S D S$ is positive if the quantization noise power is non-zero.

\section{OPTIMIZATION BASED APPROACH FOR REMOVING THE BLOCKING EFFECTS}

Based on the above observations, we propose a new approach for reducing the blocking effect by minimizing the MSDS. In our proposed method, we find a DCT coefficient set $\hat{A}_{i, j}$ subject to upper and lower limits of quantization constraints so as to minimize the expected value of the MSDS. The proposed operations are carried out only in the decoder side and therefore there is no need for transmission of additional information. We can formulate our approach as the following minimization problem:

$$
\text { minimize } \sum_{i, j \in \text { all blocks }} \varepsilon_{i j}^{2}\left(\hat{A}_{i, j}\right) .
$$

Since the above minimization problem is too computation intensive, we minimize the MSDS for each block sequentially. Before explaining our proposed approach, recall from the previous section that, the minimization of the MSDS in the $(i, j)$ th block is carried out by using the intensity values of the neighboring pixels in the adjacent blocks $(i, j-1),(i-1, j)$, $(i, j+1)$ and $(i+1, j)$. If we choose the sequence of the process to be from left to right and from top to bottom, we can use optimized intensity values in the left and upper blocks, i.e., $(i-1, j)$ th and $(i, j-1)$ th blocks, and non optimized intensity values in the right and below blocks, i.e., $(i+1, j)$ th and $(i, j+1)$ th blocks, for the optimization of the $(i, j)$ th block. This sequence is shown in Fig. 7. 
In the proposed method, we optimize $M(0<M<N)$ low frequency DCT coefficients so as to minimize the MSDS. In the remainder of this section, we show that the MSDS can be expressed as a quadratic function of the $M$ dimensional optimization coefficient vector.

First, $N$ dimensional optimization DCT coefficient vector $\hat{A}_{i, j}$ and $N$ dimensional DCT operator vector $W_{i, j}$ are divided into $M$ and $N-M$ dimensional vectors as

$$
\begin{aligned}
\hat{A}_{i, j} & =\left[\hat{A}_{L i, j}: A_{H i, j}\right]^{T} \\
W(k, l) & =\left[W_{L}(k, l): W_{H}(k, l)\right]^{T}
\end{aligned}
$$

where $W(k, l)$ is a $N^{2}$ dimensional array which is composed of IDCT operators on zigzag frequency as

$$
\begin{aligned}
W(k, l)= & {[w(0, k, l), w(1, k, l)} \\
& \left.\cdots w(\nu, k, l) \cdots w\left(N^{2}-1, k, l\right)\right]^{T}
\end{aligned}
$$

with

$$
w(\nu, k, l)=w_{I D C T}\left(F_{u}(\nu), k\right) w_{I D C T}\left(F_{v}(\nu), l\right)
$$

where the relation between horizontal frequency $u$ and vertical frequency $v$ and zigzag frequency $\nu$ are defined by functions $F_{u}$ and $F_{v}$ given by

$$
u=F_{u}(\nu) \quad v=F_{v}(\nu) .
$$

Then, an intensity $x_{i, j}(k, l)$ is obtained by

$$
x_{i, j}(k, l)=W_{i, j}^{T}(k, l) A_{i, j} .
$$

By substituting (7) into the above equation, we obtain a new expression for the intensity as

$$
x_{i, j}(k, l)=W_{L}^{T}(k, l) \hat{A}_{L i, j}+W_{H}^{T}(k, l) A_{H i, j} .
$$

Substituting the above in (5), the MSDS value along the boundary between $(i, j-1)$ th and $(i, j)$ th blocks is expressed as a function of optimizing the DCT coefficient vector as

$$
\varepsilon_{F i j}^{2}=\sum_{k=0}^{N-1}\left[\frac{1}{2}\left(3 W_{L}^{T}(k, 0)-W_{L}^{T}(k, 1)\right) \hat{A}_{L i, j}+x_{F k}\right]^{2}
$$

where

$$
\begin{aligned}
x_{F k}= & \frac{1}{2}\left[3 W_{H}^{T}(k, 0)-W_{H}^{T}(k, 1)\right) A_{H i, j} \\
& -\frac{1}{2}\left(3 x_{i, j-1}(k, N-1)\right. \\
& \left.-x_{i, j-1}(k, N-2)\right] .
\end{aligned}
$$

Similarly, the MSDS values along the remaining three boundaries are expressed as

$$
\begin{aligned}
\varepsilon_{B i j}^{2}= & \sum_{k=0}^{N-1}\left[\frac { 1 } { 2 } \left(3 W_{L}^{T}(0, k)\right.\right. \\
& \left.\left.-W_{L}^{T}(1, k)\right) \hat{A}_{L i, j}+x_{B k}\right]^{2} \\
\varepsilon_{C i j}^{2}= & \sum_{k=0}^{N-1}\left[\frac { 1 } { 2 } \left(3 W_{L}^{T}(k, N-1)\right.\right. \\
& \left.\left.-W_{L}^{T}(k, N-2)\right) \hat{A}_{L i, j}+x_{C k}\right]^{2} \\
\varepsilon_{D i j}^{2}= & \sum_{k=0}^{N-1}\left[\frac { 1 } { 2 } \left(3 W_{L}^{T}(N-1, k)\right.\right. \\
& \left.\left.-W_{L}^{T}(N-1, k)\right) \hat{A}_{L i, j}+x_{D k}\right]^{2}
\end{aligned}
$$

where

$$
\begin{aligned}
x_{B k}= & \frac{1}{2}\left[3 W_{H}^{T}(0, k)-W_{H}^{T}(1, k)\right] A_{H i, j} \\
& -\frac{1}{2}\left[3 x_{i-1, j}(N-1, k)-x_{i-1, j}(N-2, k)\right] \\
x_{C k}= & \frac{1}{2}\left[3 W_{H}^{T}(k, N-1)-W_{H}^{T}(k, N-2)\right] A_{H i, j} \\
& -\frac{1}{2}\left[3 x_{i, j+1}(k, 0)-x_{i, j+1}(k, 1)\right] \\
x_{D k}= & \frac{1}{2}\left[3 W_{H}^{T}(N-1, k)-W_{H}^{T}(N-1, k)\right] A_{H i, j} \\
& -\frac{1}{2}\left[3 x_{i+1, j}(0, k)-x_{i+1, j}(1, k)\right] .
\end{aligned}
$$

Since (9) and (11) are quadratic in the low frequency DCT coefficients $\hat{A}_{L i j}$, we can minimize the MSDS by solving the following quadratic programming problem under the constraints of the quantization upper limit vector $A_{U i j}$ and lower limit vector $A_{L i j}$ :

$$
\operatorname{minimize} \quad \hat{A}_{L i j}^{T} Q \hat{A}_{L i j}+\hat{A}_{L i j}^{T} C
$$

subject to

$$
A_{L i j} \leq \hat{A}_{L i j} \leq A_{U i j} .
$$

In (13) $Q$ is a $M \times M$ matrix and $C$ is $M$ dimensional vector given as

$$
\begin{aligned}
Q & =Q_{L F}+Q_{L B}+Q_{L C}+Q_{L D} \\
C & =-2\left(X_{F}^{T} R_{F}+X_{B}^{T} R_{B}+X_{C}^{T} R_{C}+X_{D}^{T} R_{D}\right)^{T} \\
Q_{L F} & =R_{F}^{T} R_{F}, X_{F}=\left[x_{F 0}, x_{F 1}, \cdots, x_{F k}, \cdots, x_{F N-1}\right]^{T} \\
R_{F} & =\left[W_{L F}(0): \cdots: W_{L F}(k): \cdots: W_{L F}(N-1)\right]^{T}(15)
\end{aligned}
$$

$Q_{L B}, Q_{L C}, Q_{L D}$ are defined similar to $Q_{L F} ; X_{B}, X_{C}, X_{D}$ are defined similar to $X_{F} ; R_{B}, R_{C}, R_{D}$ are $M \times N$ matrices defined similar to $R_{F}$, and

$$
\begin{aligned}
& W_{L F}(k)=\left[3 W_{L}^{T}(k, 0)-W_{L}^{T}(k, 1)\right] / 2 \\
& W_{L B}(k)=\left[3 W_{L}^{T}(0, k)-W_{L}^{T}(1, k)\right] / 2 \\
& W_{L C}(k)=\left[3 W_{L}^{T}(k, N-1)-W_{L}^{T}(k, N-2)\right] / 2 \\
& W_{L D}(k)=\left[3 W_{L}^{T}(N-1, k)-W_{L}^{T}(N-1, k)\right] / 2 .
\end{aligned}
$$

Having formulated a QP problem with linear constraints, we can now apply a variety of existing numerical techniques to solve it. We have chosen the gradient projection method to solve our QP problem [5].

\section{EXPERIMENTAL RESULTS}

To examine the effect of the proposed approach, we apply the optimization method to the image in Fig. 8(a), where only the DC and two near DC DCT coefficients are quantized to 5 and $4 \mathrm{~b}$, respectively, and the remaining coefficients are left unquantized. As seen, the blocking effect in the image in Fig. 8(a) is serious. To remove the blocking effect, we optimized the three lowest DCT coefficients so as to minimize the MSDS. The resulting picture is shown in the Fig. 8(b). As seen, the blocking effect is completely removed and the optimized image looks very similar to the original image shown in Fig. 1.

It is possible to remove the blockiness in Fig. 8(a) by traditional low-pass filtering; however, we have found that removing the blockiness in smooth areas such as the cheeks results in excessive blurring in the rest of the image. We 


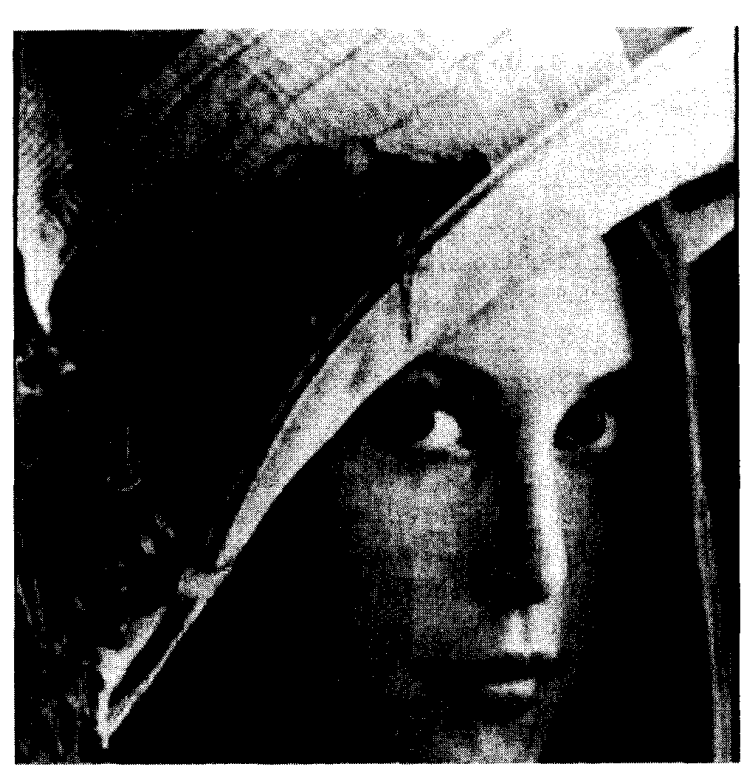

(a)

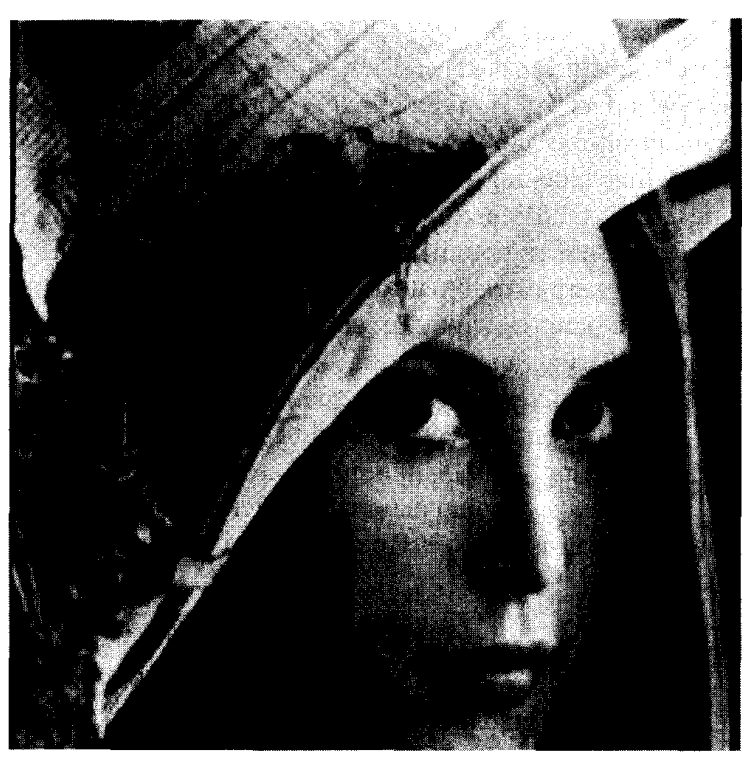

(b)

Fig. 8. (a) DC and near-DC low frequency coefficients corresponding to $(u, v)=(0,0),(u, v)=(0,1)$ and $(u, v)=(1,0)$ are quantized; high frequency DCT coefficients are not quantized. (b) Optimization is carried out for the three low frequency DCT coefficients of quantized image shown in (a).

have also found experimentally that unlike low frequency coefficients, low-pass filtering can be quite effective in dealing with blockiness caused by high frequency coefficients. This can be explained by considering that the high frequency quantization noise is small in smooth parts of the image, which are also the most noticeable.

We have also applied our optimization method to the JPEG quantized $0.27 \mathrm{~b} /$ pixel image shown in Fig. 2. The result

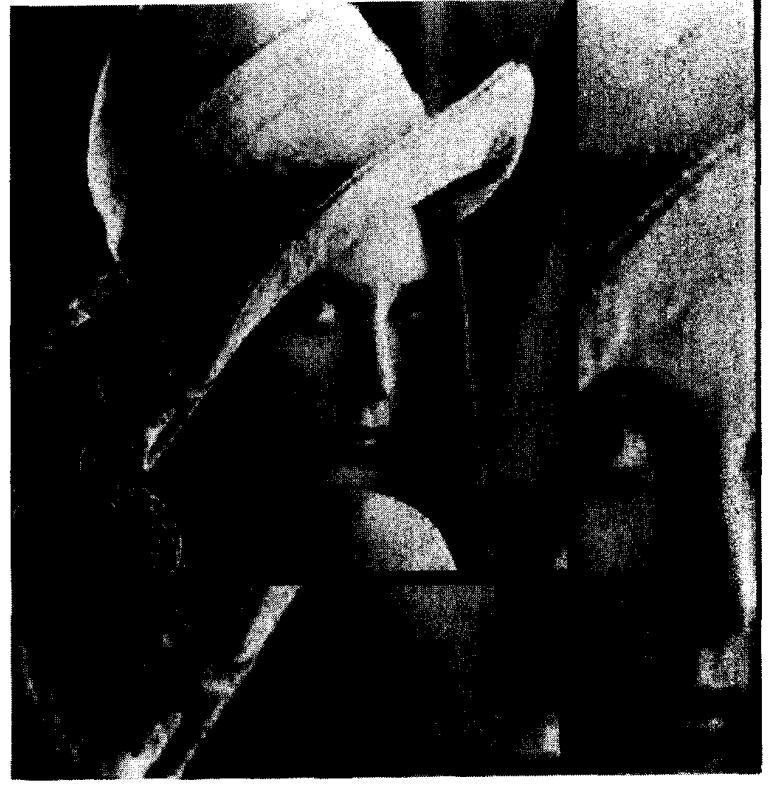

Fig. 9. The optimization is carried out for three low frequency DCT coefficients of the JPEG quantized image shown in Fig. 2. Since high frequency components are not optimized, there is blocking effect around hat area.

is shown in Fig. 9 where three lower DCT coefficients are optimized. Since the number of optimized coefficients in Fig. 9 is only 3, we still notice the blocking effects in Lena's hat area which is caused by the quantization of the higher frequency DCT coefficients.

To enhance the merits of the low-pass filtering and the proposed optimization method, we have combined the two methods, where the low-pass filtering is carried out after the three coefficient optimization. We used the following low-pass filter from [2]:

$$
\begin{aligned}
h\left(n_{1}, n_{2}\right) & =h\left(n_{1}\right) h\left(n_{2}\right) \\
h( \pm 2) & =0.1 \quad h( \pm 1)=0.24 \quad h(0)=0.32
\end{aligned}
$$

and the resulting image is shown in Fig. 10(a). As shown, the picture results in better blocking effect reduction and picture quality than that of the three coefficient optimization shown in Fig. 9, or the anisotropic filtering of Fig. 4, or the plain lowpass filtering of the JPEG quantized image of Fig. 2, shown in Fig. 10(b). The reason for this is that the blocking effect due to low frequency coefficients is removed well by the three coefficient optimization and the high frequency blocking effect is removed by the low-pass filtering method.

The computational complexity of this approach is quite small: it can be shown that if the number of zonally coded coefficients is $M_{L}$, the number of optimized coefficients is $M$ and the block size is $N \times N$, then the total number of multiplications for the quadratic optimization of each block is $4\left(M_{L}-M\right)+M N+M$. For $N=8, M_{L}=10$ and $M=3$ this translates into 55 multiplications per block, which is quite reasonable. 


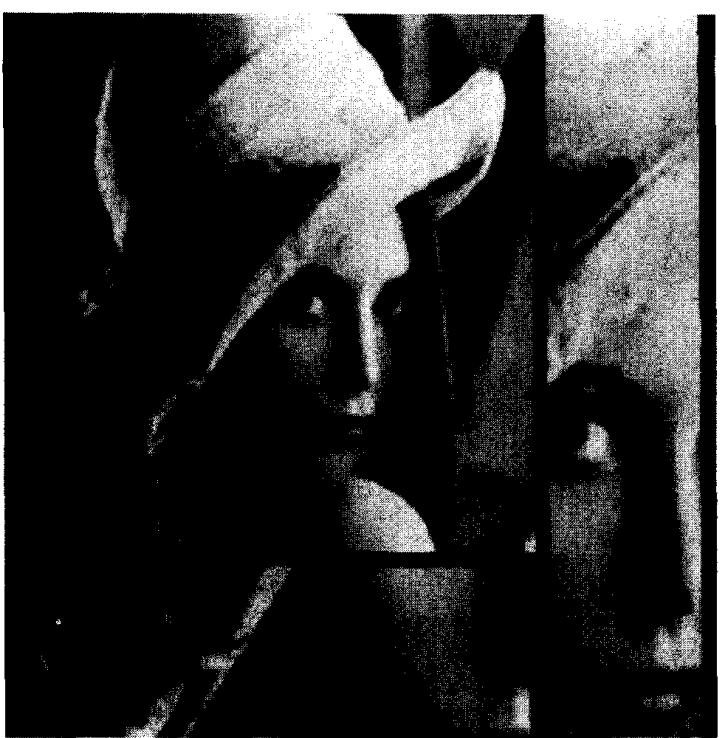

(a)

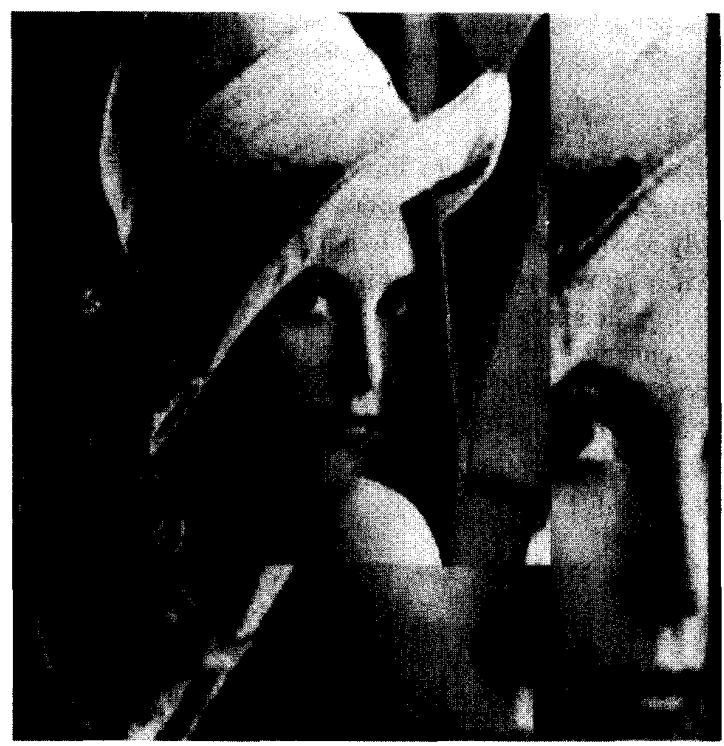

(b)

Fig. 10. (a) The optimization is carried out for three low frequency DCT coefficients of the JPEG quantized image in Fig. 2, then traditional low-pas filtering is applied to the optimized image. (b) Separable low-pass filtering applied to the JPEG quantized image in Fig. 2.

As shown in Appendix, the expected value of MSDS increases due to quantization. This can actually be observed in the case of the JPEG quantization. As seen in Table I, the MSDS increase from the original picture in Fig. 1 to the quantized picture in Fig. 2 is a positive value, i.e., 9563. the MSDS increase after applying some blocking effect reduction methods is also shown in the table. As seen, the anisotropic low-pass filter approach and the combination approach show large MSDS reductions. The optimization approach with 3 and
TABLE I

MSDS INCREASE AND SNR RATIO FOR VARIOUS IMAGFS

\begin{tabular}{|l|c|c|}
\hline & MSDS Increase & SN \\
\hline JPEG Quantization (Fig. 2) & 9563 & $27.5 \mathrm{~dB}$ \\
\hline LPF Approach (Fig. 4) & -1607 & $28.3 \mathrm{~dB}$ \\
\hline AC Prediction (Fig. 5) & 8614 & $27.2 \mathrm{~dB}$ \\
\hline Optimization 3 Coef. (Fig.9) & 6024 & $27.3 \mathrm{~dB}$ \\
\hline Optimization 6 Coef (Fig.13) & 4672 & $27.3 \mathrm{~dB}$ \\
\hline Combination Method (Fig.10 -a) & -2806 & $27.6 \mathrm{~dB}$ \\
\hline
\end{tabular}

6 coefficient optimization shows moderate reduction of the MSDS. However, since the effects of the MSDS reduction are concentrated in the low frequency component of the picture, subjective visual quality should be given more importance than MSDS reduction.

\section{CONCLUSION}

This paper has presented a new approach for reducing the blocking effect. A new criterion, MSDS, which is a measure of blocking effect was proposed; it was shown that the expected value of the MSDS increases after quantizing the DCT coefficients. Therefore, our approach removes the blocking effect by minimizing the MSDS, while imposing linear constraints corresponding to quantization bounds. We showed that blocking effect due to quantization of the DC or near DC DCT coefficients is removed well by solving the QP problem to optimize the DCT coefficients. Computer simulations were carried out to evaluate the proposed method, and to compare it with the conventional and anisotropic low-pass filtering approach and AC prediction method for the JPEG coding. The highest quality image was obtained by a combination of MSDS minimization and subsequent low-pass filtering. MSDS minimization for small number of DCT coefficients is computationally efficient and requires no additional information to be transmitted to the receiver.

\section{APPENDIX}

\section{EXPECTED VALUE OF THE MSDS}

The boundary gradients are a linear function of the DCT coefficients and can be expressed as

$$
d_{i, j}^{M F}(k)=W^{T}(k, 0) A_{i, j}-W^{T}(k, N-1) A_{i, j-1}
$$

with, $d_{i, j}^{M B}(k), d_{i, j}^{M C}(k)$, and $d_{i, j}^{M D}(k)$ defined in a similar fashion. Similarly, the interpolated gradients are linear in terms of the DCT coefficients and are given by

$$
\begin{aligned}
\bar{d}_{i, j}^{F}(k)= & \left\{\left[W^{T}(k, 1)-W^{T}(k, 0)\right] A_{i, j}\right. \\
& +\left[W^{T}(k, N-1)\right. \\
& \left.\left.-W^{T}(k, N-2)\right] A_{i, j-1}\right\} / 2
\end{aligned}
$$

with $\bar{d}_{i, j}^{B}(k), \bar{d}_{i, j}^{C}(k), \bar{d}_{i, j}^{D}(k)$ defined in a similar fashion. The mean square difference along the block boundary between $(i, j-1)$ th and $(i, j)$ th blocks is expressed in terms of the 
DCT coefficients by substituting (17) and (18) into (5) as

$$
\begin{aligned}
\varepsilon_{F i j}^{2} & =\sum_{k=0}^{N-1}\left[W_{F}^{T}(k) A_{i, j}+W_{\mathcal{B}}^{T}(k) A_{i, j-1}\right]^{2} \\
W_{F}(k) & =[W(k, 1)-3 W(k, 0)] / 2 \\
W_{B}(k) & =[W(k, N-2)-3 W(k, N-1)] / 2 .
\end{aligned}
$$

Similar expressions for $\varepsilon_{B i j}^{2}, \varepsilon_{C i j}^{2}$, and $\varepsilon_{D i j}^{2}$ can be obtained. Using matrix notion, these mean square differences can be written as:

$$
\begin{aligned}
& \varepsilon_{F i j}^{2}=A_{i, j}^{T} Q_{F} A_{i, j}+A_{i, j}^{T} Q_{B} A_{i, j-1}+2 A_{i, j}^{T} Q_{F B} A_{i, j-1} \\
& \varepsilon_{B i j}^{2}=A_{i, j}^{T} Q_{C} A_{i, j}+A_{i, j}^{T} Q_{D} A_{i-1, j}+2 A_{i, j}^{T} Q_{C D} A_{i-1, j} \\
& \varepsilon_{C i j}^{2}=A_{i, j}^{T} Q_{F} A_{i, j}+A_{i, j}^{T} Q_{B} A_{i, j+1}+2 A_{i, j}^{T} Q_{F B} A_{i, j+1} \\
& \varepsilon_{D i j}^{2}=A_{i, j}^{T} Q_{C} A_{i, j}+A_{i, j}^{T} Q_{D} A_{i+1, j}+2 A_{i, j}^{T} Q_{C D} A_{i+1, j}
\end{aligned}
$$

where

$$
\begin{aligned}
Q_{F B}= & R_{F} R_{B}^{T}, \quad Q_{C D}=R_{C} R_{D}^{T}, \quad Q_{F}=R_{F} R_{F}^{T} \\
R_{F}= & {\left[W_{F}(0): W_{F}(1): \cdots\right.} \\
& \left.W_{F}(k): \cdots: W_{F}(N-1)\right]^{T}
\end{aligned}
$$

$Q_{B}, Q_{C}, Q_{D}$ are defined similar to $Q_{F}$ and $R_{B}, R_{C}, R_{D}$ are defined similar to $R_{F}$. The expected value of the MSDS is obtained for two cases of quantized and unquantized DCT coefficients. By taking statistical average of MSDS in (19), we obtain expected values of the MSDS as

$$
\begin{aligned}
E\left[\varepsilon_{F i j}^{2}\right]= & E\left[A_{i, j}^{T} Q_{F} A_{i, j}\right]+E\left[A_{i, j-1}^{T} Q_{B} A_{i, j-1}\right] \\
& +2 E\left[A_{i, j}^{T} Q_{F B} A_{i, j-1}\right] \\
E\left[\varepsilon_{B i j}^{2}\right]= & E\left[A_{i, j}^{T} Q_{C} A_{i, j}\right]+E\left[A_{i-1, j}^{T} Q_{D} A_{i-1, j}\right] \\
& +2 E\left[A_{i, j}^{T} Q_{C D} A_{i, j-1}\right] \\
E\left[\varepsilon_{C i j}^{2}\right]= & E\left[A_{i, j}^{T} Q_{F} A_{i, j}\right]+E\left[A_{i, j}^{T} Q_{B} A_{i, j+1}\right] \\
& +2 E\left[A_{i, j}^{T} Q_{F B} A_{i, j+1}\right] \\
E\left[\varepsilon_{D i j}^{2}\right]= & E\left[A_{i, j}^{T} Q_{C} A_{i, j}\right]+E\left[A_{i, j}^{T} Q_{D} A_{i+1, j}\right] \\
& +2 E\left[A_{i, j}^{T} Q_{C D} A_{i+1, j}\right] .
\end{aligned}
$$

Also, the MSDS after quantization is given by

$$
\begin{aligned}
\tilde{\varepsilon}_{F i j}^{2}= & \tilde{A}_{i, j}^{T} Q_{F} \tilde{A}_{i, j}+\tilde{A}_{i, j}^{T} Q_{B} \tilde{A}_{i, j-1} \\
& +2 \tilde{A}_{i, j}^{T} Q_{F B} \tilde{A}_{i, j-1}
\end{aligned}
$$

with $\hat{\varepsilon}_{B i j}^{2}, \tilde{\varepsilon}_{C i j}^{2}$, and $\tilde{\varepsilon}_{D i j}^{2}$ defined in a similar fashion. In (23), $\tilde{A}_{i, j}$ is a DCT coefficient vector for $(i, j)$ th block after quantization and is given by adding a quantization noise vector $V_{i, j}$ to the original DCT coefficient vector $A_{i, j}$ as

$$
\tilde{A}_{i, j}=A_{i, j}+V_{i, j} \text {. }
$$

Each component of $V_{i j}$ is assumed to be an independent random variable uniformly distributed between quantization upper and lower bounds. From the assumption of the statistical independence between the DCT coefficients and from (22),
(23), and (24), the expected value of the MSDS values after quantization becomes

$$
\begin{aligned}
E\left[\tilde{\varepsilon}_{F i j}^{2}\right]= & E\left[\varepsilon_{F i j}^{2}\right]+E\left[V_{i, j}^{T} Q_{F} V_{i, j}\right]+E\left[V_{i, j-1}^{T} Q_{B} V_{i, j-1}\right] \\
& +2 E\left[V_{i, j}^{T} Q_{F B} V_{i, j-1}\right]
\end{aligned}
$$

with $E\left[\tilde{\varepsilon}_{B i j}^{2}\right], E\left[\tilde{\varepsilon}_{C i j}^{2}\right], E\left[\tilde{\varepsilon}_{D i j}^{2}\right]$ defined in a similar fashion. Since quantization noise components are statistically independent, the forth term in (25) is zero; we can rewrite (25) by using only $(i, j)$ th noise vector because statistical characteristics of the noise components are identical:

$$
E\left[\tilde{\varepsilon}_{F i j}^{2}\right]=E\left[\varepsilon_{F i j}^{2}\right]+E\left[V_{i, j}^{T}\left(Q_{F}+Q_{B}\right) V_{i, j}\right]
$$

with $E\left[\tilde{\varepsilon}_{B i j}^{2}\right], E\left[\tilde{\varepsilon}_{C i j}^{2}\right], E\left[\tilde{\varepsilon}_{D i j}^{2}\right]$ defined similarly. From (26), we obtain the expected value of the MSDS increase due to quantization, $E[\delta M S D S]$ to be:

$$
\begin{aligned}
E[\delta M S D S] & =E\left[V_{i, j}^{T} Q V_{i, j}\right] \\
Q & =2\left(Q_{F}+Q_{B}+Q_{C}+Q_{D}\right) .
\end{aligned}
$$

Since $Q_{F}, Q_{B}, Q_{C}$, and $Q_{D}$ are all non-negative symmetric matrices shown in (20), the matrix $Q$ is also non-negative symmetric. If we define a regular matrix $G$, and vector $\tilde{V}_{i, j}$ :

$$
G^{T} Q G=\operatorname{diag}\left[\lambda_{0}, \cdots, \lambda_{i}, \lambda_{M}\right]^{T}, \quad V_{i, j}=G \tilde{V}_{i, j}
$$

with $\lambda_{i}$ being the $i$ th eigenvalue of the matrix $Q$, then the expected value of the MSDS increase is given by

$$
E[\delta M S D S]=\sum_{k=0}^{M-1} \lambda_{k} \tilde{\sigma}_{k}^{2}
$$

where $\tilde{\sigma}_{k}$ is the variance of the $k$ th element of $\tilde{V}_{i, j}$ given by $\tilde{\sigma}_{k}=g_{k}^{T} v$ with $g_{k}$ being the $k$ th row of the matrix $G$ and $v$ being the vector consisting of the variance of the elements of vector $V_{i, j}$. From the definition of the matrix $Q, \lambda_{i}$ is non-negative for $0 \leq i \leq M-1$ and positive at least for one $i$. Similarly, since at least one component of $g_{i}$ is nonzero (because $G$ is regular matrix), the expected value of the MSDS increase becomes positive if all DCT coefficients are quantized.

\section{REFERENCES}

[1] H. Reeve and J. S. Lim, "Reduction of blocking effects in image coding," Opt. Eng., vol. 23, no. 1, pp. 34-37, Jan. 1984.

[2] K.-H. Tzou, "Post-filtering of transform-coded images," in Applications of Digital Image Process. XI Proc. SPIE, vol. 974, 1988, pp. 121-126.

[3] JPEG Committee Draft of the ISO/IEC JTC1 COM VIII-R.24.

[4] W. B. Pennebaker and J. L. Mitchell, JPEG Still Image Compression Standard. New York: Van Nostrand Reinhold, 1992.

[5] D. G. Luenberger, Linear and Nonlinear Programming. Reading, MA: Addison-Wesley, 1984. 


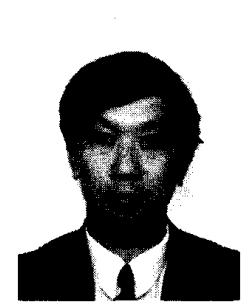

Shigenobu Minami was born in Wakayama, Japan, on May 6, 1954. He received the B.E. and M.E. degrees in electronic engineering from the Wasada University, Shinjuku, Tokyo, Japan, in 1977 and 1979 , respectively.

After joining the Research and Development Center, Toshiba Corporation, Kawasaki, in 1979, he was engaged in research in digital signal processing applications, such as echo canceler. From 1989 to 1991 , he was a visiting scientist with the EECS University of California, Berkeley. He is currently a manager in the Information and Communication Systems Laboratory, Toshiba Corporation. His current interests include the speech and video signal processing for communications, DSP applications and architectures.

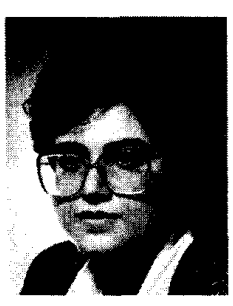

Avideh Zakhor received the B.S. degree from the California Institute of Technology, Pasadena, and the S.M. and Ph.D. degrees from the Massachusetts Institute of Technology, Cambridge, all in electrical engineering, in 1983, 1985, and 1987, respectively.

In 1988, she joined the Faculty at the University of California, Berkeley, where she is currently Associate Professor with the Department of Electrical Engineering and Computer Sciences. Her research interests are in the general area of signal processing and its applications to images and video, and biomedical data. She has been a consultant to a number of industrial organizations and holds four US patents.

Dr. Zakhor was a General Motors scholar from 1982 to 1983, received the Henry Ford Engineering Award and Caltech Prize in 1983, was a Hertz Fellow from 1984 to 1988, received the Presidential Young Investigators (PYI) award, IBM junior faculty development award, and Analog Devices junior faculty development award in 1990, and Office of Naval Research (ONR) young investigator award in 1992. She is a member of the technical committee for multidimensional digital signal processing. 